\title{
An assessment of $J / \psi$ formation in the light of initial RHIC data
}

\author{
R. L. Thews \\ Department of Physics, University of Arizona, Tucson, AZ 85721, USA
}

\begin{abstract}
Predictions of $J / \psi$ formation at RHIC via "off-diagonal" combinations of charm and anticharm quarks in a region of color deconfinement are confronted with initial data from the PHENIX collaboration. We find that the measured centrality behavior places significant constraints on the various parameters which control model calculations of $J / \psi$ formation. Within present statistical and systematic uncertainties, one can map out a region of parameter space within which the contribution of formation in a deconfined phase is allowed. As these uncertainties decrease and new data from d$\mathrm{Au}$ interactions becomes available, it is expected that definitive tests for the presence of this formation mechanism will be possible. We anticipate that the rapidity and transverse momentum spectra will prove decisive for a final determination.
\end{abstract}

\section{Introduction}

Initial data at RHIC energy on $J / \psi$ production in $\mathrm{Au}-\mathrm{Au}$ collisions has been eagerly awaited, in terms of a signal for the presence of color deconfinement [1]. Of special interest is the possibility that a direct extrapolation of anomalous suppression from the SPS energy range could be supplanted by a new formation mechanism fueled by the presence of multiple pairs of charm quarks in each nuclear collision at sufficiently high energy [2]. One can argue on general grounds that the resulting $J / \psi$ formation will grow quadratically with the total number of unbound charm quark pairs, with a normalization factor which depends on the specific formation mechanism [3]. Predictions for this type of formation have been made for two specific models. The statistical hadronization model [4] assumes that at the time of hadronization, charm quarks are distributed into hadrons according to statistical rules, incorporating an additional fugacity factor to conserve charm. The kinetic formation model [5, 6] considers $J / \psi$ formation within the region of deconfinement, and calculates the net number remaining at hadronization due to a competition between formation and breakup reactions. In this work, we confront the kinetic formation model predictions with the initial data from the PHENIX collaboration at RHIC. This initial data [7, 8] consists of a measurement of $J / \psi$ produced at central rapidity in $\mathrm{Au}-\mathrm{Au}$ collisions at $\sqrt{s}=200 \mathrm{GeV}$, presented in three centrality bins. In addition, there is data on $J / \psi$ production in pp collisions in three rapidity bins. There is also an indirect measurement of initial charm production via detection of high- $p_{t}$ electrons [9]. 
In this presentation we first give in the next section a brief summary of the kinetic model formulation, with a discussion of primary uncertainties and ranges of parameters. The following section is devoted to a comparison of the predictions of this model with the initial PHENIX numbers, both the absolute values and the centrality dependence. Finally, we explore the regions of parameter space which are consistent with the central values of the initial data, and comment on aspects for the future.

\section{Kinetic formation model}

The kinetic formation model is most easily motivated in a scenario where suppression of $J / \psi$ due to deconfined color is related to their breakup via collisions with gluons [10]. Given the distribution of gluons and the cross section for this reaction, one can calculate the rate for destruction of $J / \psi$ in the region of deconfinement. It is then clear that one should also take into account the inverse reaction, in which charm and anticharm quarks interact to form $J / \psi$ with emission of a gluon. The net effect is a competition between these two processes, which one can express via a Boltzmann equation for the charm quark and $J / \psi$ populations.

$$
\frac{d N_{J / \psi}}{d t}=\lambda_{\mathrm{F}} N_{c} \rho_{\bar{c}}-\lambda_{\mathrm{D}} N_{J / \psi} \rho_{g}
$$

where $\rho$ denotes number density, and the reactivity $\lambda$ is the reaction rate $\left\langle\sigma v_{\text {rel }}\right\rangle$ averaged over the momentum distribution of the initial participants, i.e. $c$ and $\bar{c}$ for $\lambda_{F}$ and $J / \psi$ and $g$ for $\lambda_{D}$. In our calculations, this equation is solved numerically while enforcing exact charm conservation. At SPS energy, one finds that the formation process is negligble, since the average number of charm quark pairs produced even in central collisions is much smaller than unity. At high energy where one expects the number of initial charm pairs $N_{c \bar{c}}$ will become large, one can find an approximate analytic solution for Eq. 11 which exhibits the anticipated quadratic dependence.

$$
N_{J / \psi}(t)=\epsilon(t) \times\left[N_{J / \psi}\left(t_{0}\right)+N_{c \bar{c}}^{2} \int_{t_{0}}^{t} \lambda_{\mathrm{F}}\left[V\left(t^{\prime}\right) \epsilon\left(t^{\prime}\right)\right]^{-1} d t^{\prime}\right]
$$

where $\epsilon(t)=e^{-\int_{t_{0}}^{t} \lambda_{\mathrm{D}} \rho_{g} d t}$ would be the equilvalent suppression factor in this scenario if the formation mechanism were neglected. Here we have assumed the densities are uniform within a deconfinement volume $V(t)$. We use a thermal gluon density, initial temperature $T_{0}$ as a variable parameter, and fixed final deconfinement temperature. The deconfinement volume is assumed to expand isentropically, for scenarios in which transverse expansion is controlled by a parameter vtr. The initial population of $J / \psi$ is taken to be a fraction $x$ of the initial number of charm quark pairs. The cross section is taken from an OPE-based model, based on the color dipole interaction of a nonrelativistic bound state with a coulomb bound state spectrum. For the centrality dependence, we utilize a calculation of the number of participants as a function of impact parameter. We also calculate the participant density in the transverse plane, and define an effective transverse area as the ratio of total to density. The participant 
density is also used to specify the centrality dependence of initial temperature $T_{0}$. All centrality-dependent quantities are then scaled to their $b=0$ values.

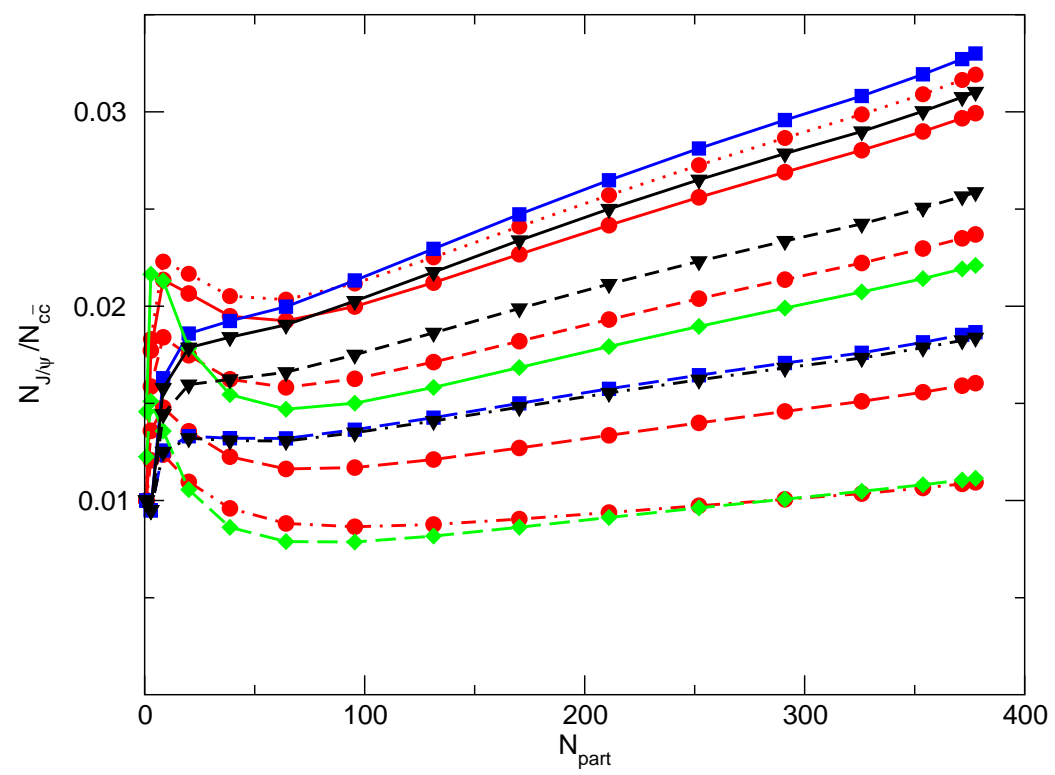

Figure 1. Predictions for $J / \psi$ formation. All use $N_{c \bar{c}}=10$ and $\mathrm{x}=0.01$.

Our initial calculations were performed for $T_{0}=0.3,0.4$, and $0.5 \mathrm{GeV}, \mathrm{x}=0.01$, and $N_{c \bar{c}}(\mathrm{~b}=0)=10$. To minimize the dependence on $N_{c \bar{c}}$, the ratio $N_{J / \psi} / N_{c \bar{c}}$ was presented. Shown in Figure 1 are predictions for this ratio as published in Ref. [2], where the legends are defined. The primary sensitivity was due to the charm quark momentum distribution. As might be expected, a thermal distribution was most efficient in formation, while distributions with various increasing rapidity widths predicted smaller numbers. All of the curves rise with increasing centrality, and have magnitudes near or above the assumed initial value $(\mathrm{x}=0.01)$.

\section{PHENIX data vs. predictions}

For an initial comparison of these predictions with the PHENIX data, one must translate the measured $d N_{J / \psi} / d y$ at $\mathrm{y}=0$ to the total $N_{J / \psi}$, and calculate the initial $N_{c \bar{c}}$ in each centrality bin. For the former, we assumed a flat $J / \psi$ rapidity distribution with an effective $\Delta y=4$. The $N_{c \bar{c}}$ for each centrality region were scaled to the $N_{c \bar{c}}(\mathrm{~b}=0)=10$ assumed in the calculated curves, and varied with centrality according to the nuclear overlap function $T_{A A}(\mathrm{~b})$ evaluated at an impact parameter corresponding to the central values of the experimental bins. The calculated ratios obtained in this way from the data are shown in Figure 2, where the error bars are taken from a sum of statistical and systematic uncertainties. One sees that the central values decrease with centrality. (This is the same behavior as shown in Reference [7] for the measured $J / \psi$ rapidity density scaled by the number of binary collisions.) It must be remembered that the ratios extracted from the experimental data in this way are only valid if the experimental value of total charm production proves to be the same as that assumed in these model 
calculations. For ease of comparison, one set of model predictions for $T_{0}=0.4 \mathrm{GeV}$ and charm quark momentum widths $\Delta y=1,2,3,4$ are shown from among those in Figure 11. Also shown are three additional model curves which use the exact charm quark momentum distribution from a LO pQCD calculation.

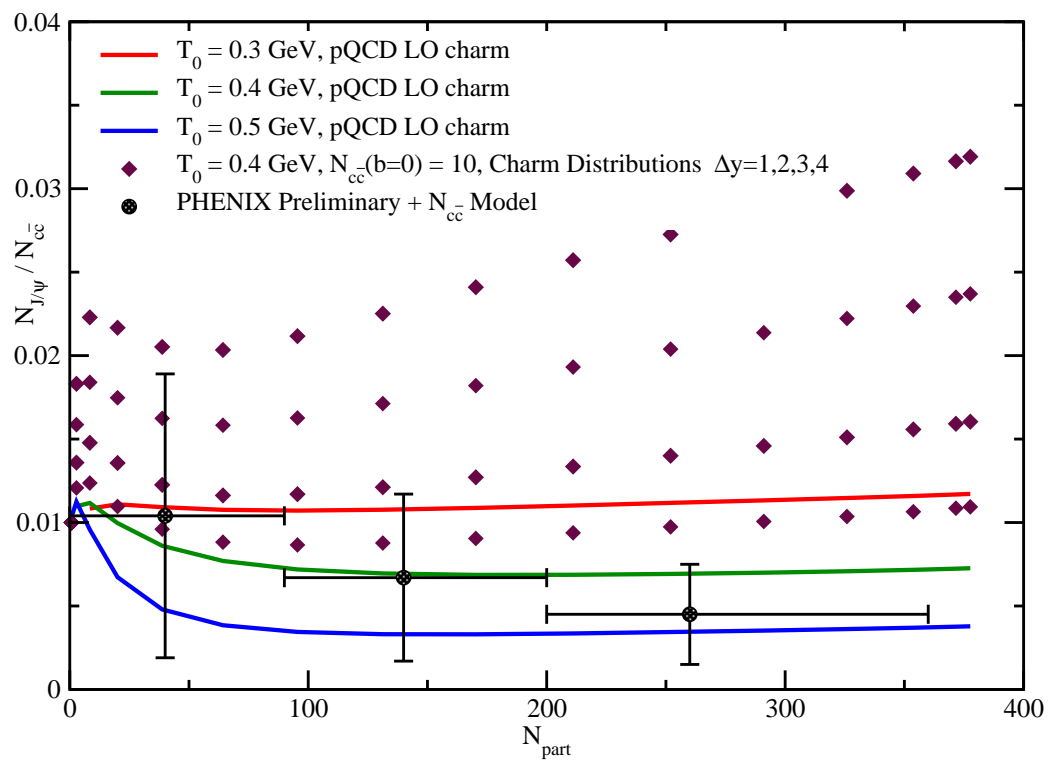

Figure 2. Indirect comparison of formation model with PHENIX data.

These latter results are in general agreement with the preliminary data. For a more systematic comparison of the data with formation model results, we will restrict the charm momentum distributions to the pQCD set.

\section{PHENIX data and formation parameters}

In this section we adapt the model calculations to compare directly with $d N_{J / \psi} / d y$ data. The calculated $N_{J / \psi}$ have one component originating from initial production and a second from the combined formation/dissociation processes. The first is converted to a rapidity density according to the measured $J / \psi$ rapidity distribution from production in pp interactions [7, and the second from a model calculation of the rapidity distribution which follows from the formation mechanism [11]. One also needs the $N_{c \bar{c}}$ values, which we estimate from preliminary open charm cross section 9. Since there is presently an uncertainty of order factor of two in this estimate, we choose to present calculations for a wide range of $N_{c \bar{c}}(\mathrm{~b}=0)=5,10,15,20$. We start with zero for our initial production parameter $x$. Given these parameter constraints, we scan over a range of $T_{0}$ to select sets which are roughly compatible with the data. We show in Figure 3 such a set. One may question how the formation model is able to produce these curves, which rise with centrality less rapidly than binary scaling in order to be compatible with the data. The answer is that the formation model involves an inverse volume, which would normally provide a linear $N_{\text {part }}$ dependence. However this volume is time dependent and is integrated over the deconfinement lifetime along with factors sensitive to the 
dissociation probability. We also extend the parameter space to include a nonzero transverse expansion of the deconfinement volume, shown in Figure 4 . One sees that there is a significant correlation between sets of allowed parameters, and that a precise experimental constraint on $N_{c \bar{c}}$, for example, would place significant constraints on the others.

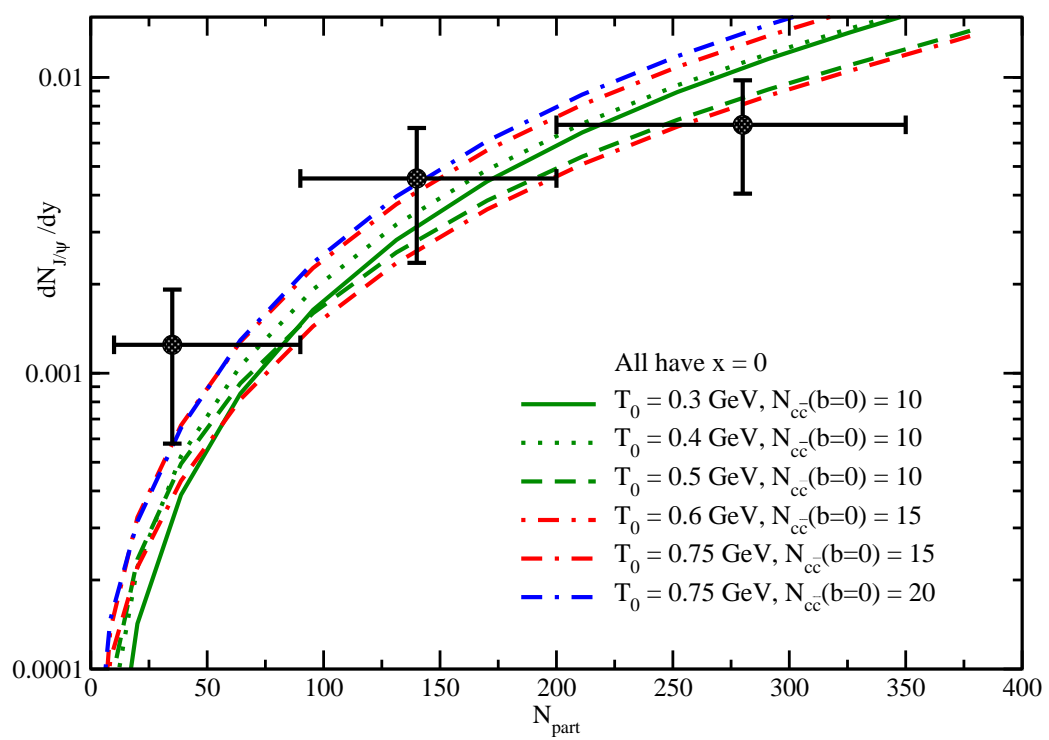

Figure 3. Kinetic model variation with $T_{0}$ and $N_{c \bar{c}}$.

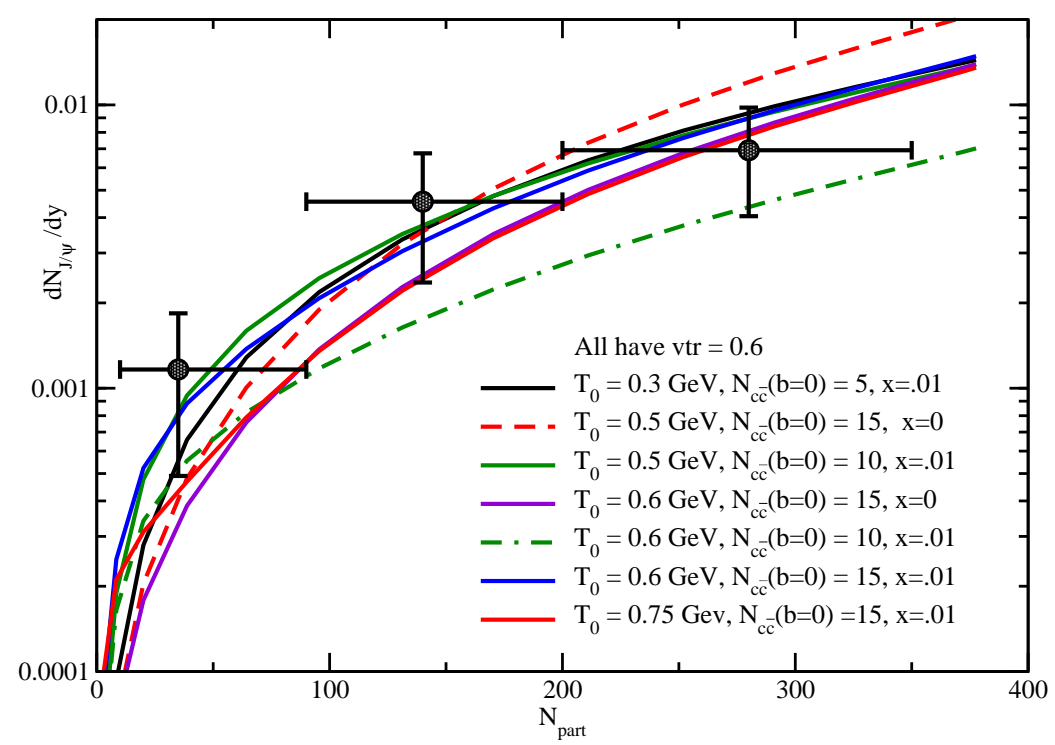

Figure 4. Kinetic model results for $\mathrm{d} N_{J / \psi} /$ dy with transverse expansion.

Finally, we show for completeness an alternate scenario in Figure 5. Along with the data and one set of formation model parameters which reproduces the central values of the measurements, we show two solutions in which only the dissociation mechanism is nonzero, using $T_{0}=0.3 \mathrm{GeV}$ and $0.4 \mathrm{GeV}$ which control the gluon density and the deconfinement lifetime. For comparison, the centrality dependence which would result 
from pure binary collision scaling are also shown. It appears that even if the uncertainties in the data points were reduced considerably, one could probably find an acceptable dissociation fit. In that case, it will be essential to compare not only the magnitudes

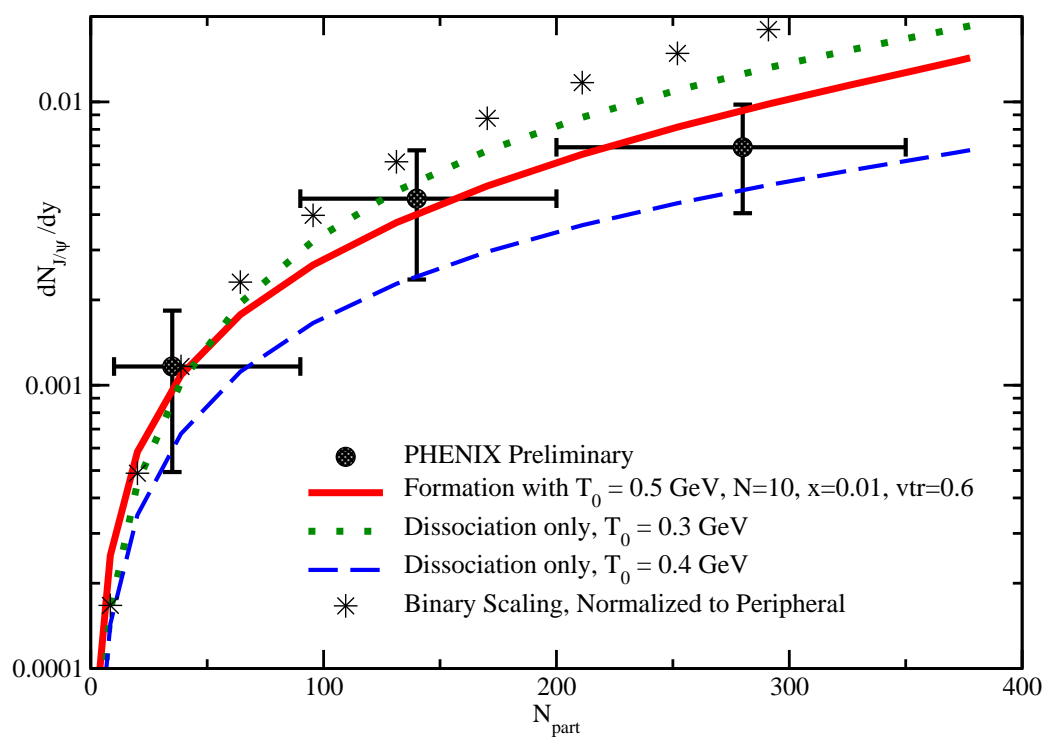

Figure 5. Comparison of formation formation calculation and binary scaling with PHENIX data.

and centrality dependence, but also to confront the different scenarios with measured $J / \psi$ rapidity and transverse momentum behavior. Work along these lines is underway.

Acknowledgment: This work was supported in part by U.S. Department of Energy Grant DE-FG03-95ER40937.

\section{References}

[1] Matsui T and Satz H 1986 Phys. Lett. B 178416

[2] Thews R L, Schroedter M and Rafelski J 2001 Phys. Rev. C 63054905 arXiv:hep-ph/0007323

[3] Thews R L 2002 Nucl. Phys. A 702341 arXiv:hep-ph/0111015

[4] Braun-Munzinger P and Stachel J 2000 Phys. Lett. B 490196 arXiv:nucl-th/0007059

[5] Thews R L 2002 Proc. Pan American Advanced Studies Institute: New States of Matter in Hadronic Interactions (PASI 2002) (AIP Conference Proceedings Volume 631) p 490 arXiv:hep-ph/0206179

[6] Thews R L and Rafelski J 2002 Nucl. Phys. A 698575 arXiv:hep-ph/0104025

[7] Frawley A D 2002 [PHENIX Collaboration], J/psi $\rightarrow e$ e and $j / p s i \rightarrow m u$ mu measurements in $A u$ Au and $p$ collisions at $s(N N)^{* *}(1 / 2)=200-G e V$ arXiv:nucl-ex/0210013

[8] Nagle J L 2002 [The PHENIX Collaboration], Leptonic Observables in Ultra-Relativistic Heavy Ion Collisions arXiv:nucl-ex/0209015

[9] Averbeck R 2002 [PHENIX Collaboration], Single leptons from heavy-flavor decays at RHIC arXiv:nucl-ex/0209016

[10] Kharzeev D and Satz H 1994 Phys. Lett. B 334155 arXiv:hep-ph/9405414

[11] Mangano M L and Thews R L work in progress 